\title{
Avaliação do estado nutricional de adolescentes, segundo o sexo
}

Sílvia Oliveira Lopes, Thais de Souza Magalhães, Fabrício da Silva Ferraz, Carina Pinto, Elizangela da Silva Miguel, Silvia Eloiza Priore

\section{Resumo}

Etapa essencial para o desenvolvimento biopsicossocial do ser humano, a adolescência é caracterizada pelas mudanças no desenvolvimento do corpo, além de ser a fase das novas descobertas. O estado nutricional é de particular interesse, pois a presença de obesidade nesta faixa etária tem relação com o aparecimento de doenças e distúrbios, algumas vezes irreversíveis. Avaliar o estado nutricional de adolescentes, segundo o sexo. Participaram do estudo estudantes do $1^{\circ}$ ano de um Colégio de Aplicação de Viçosa, MG, que realizaram avaliação antropométrica. Com auxílio de antropômetro vertical e de uma balança portátil foram aferidos estatura e peso, respectivamente. Foi calculado o índice de massa corporal (peso/altura ${ }^{2}$ ) por idade. A avaliação contou com a utilização do software WHO AnthroPlus para classificar o estado nutricional dos adolescentes. Foram utilizados para classificação os pontos de corte da World Health Organization (2007). Estabeleceu-se como ponto de referência para aferição do perímetro da cintura (PC) dois centímetros acima da cicatriz umbilical. Calculou-se a relação cintura estatura (RCE) e adotou-se como presença de risco cardiometabólico, valor de RCE $\geq 0,5$. Realizou-se análise descritiva dos dados, seguida do qui-quadrado de Pearson para verificar presença de associação. Avaliou-se 471 adolescentes de um Colégio de Aplicação, sendo 52,2\% $(n=246)$ do sexo feminino, nos anos de 2012 a 2015. Em ambos os sexos, 98\% apresentaram estatura/idade adequada. Em relação ao estado nutricional, segundo IMC/Idade, constatou-se que as meninas apresentaram maior adequação; $87,4 \%(n=215)$ eutróficas, enquanto que entre os meninos este valor foi de $72 \%(n=162)$. Indivíduos do sexo masculino obtiveram prevalências de sobrepeso e obesidade superior ao feminino, sendo $16,9 \%(n=38)$ e $7,1 \%(n=16)$, respectivamente; nas meninas os percentuais de sobrepeso e obesidade foram inferiores, correspondendo a $5,3 \%(n=13)$ e $2,8 \%(n=7)$, respectivamente. Observou-se associação entre o sexo e estado nutricional pelo IMC/I ( $\mathrm{p}=0,001)$, no entanto, entre sexo e estatura/idade e RCE não se observou associação. Adolescentes do sexo feminino obtiveram maior percentual de adequação na RCE, correspondendo a 95,5\% $(n=235)$. Adolescentes do sexo masculino apresentaram índice de inadequação elevado se comparado ao feminino, 14,2\% ( $\mathrm{n}=32)$ dos meninos apresentaram risco cardiometabólico e 4,5\% $(n=11)$ das meninas obtiveram valores de RCE acima de 0,5. Pode-se concluir que adolescentes do sexo masculino do estudo estavam mais susceptíveis ao desenvolvimento de doenças cardiovasculares, por apresentarem maior percentual de RCE alterada. Em ambas as variáveis analisadas, constatou-se que há maior adequação nos adolescentes do sexo feminino.

Descritores: Adolescentes; Estado nutricional; Antropometria. 